\title{
Use of Transesophageal Echocardiography During Implantation of Aortic Endoprosthesis (Stent). Initial Experience
}

\author{
Cláudio Henrique Fischer, Orlando Campos Fo, José Honório de Almeida Palma da Fonseca, Cláudia \\ Maria Rodrigues Alves, José Augusto Marcondes Sousa, Edgar Bezerra de Lira Fo, \\ Antonio Carlos de Camargo Carvalho, Ângelo Amato Vicenzo de Paola, Ênio Buffolo \\ São Paulo, SP - Brazil
}

\begin{abstract}
Objective - To report the role played by transesophageal echocardiography during implantation of self-expanding aortic endoprostheses (stent) at a hemodynamics laboratory.
\end{abstract}

Methods - Thirteen patients underwent stent implantation in the descending thoracic aorta with the aid of transesophageal echocardiography during the entire procedure. Indications for stenting were as follows: 8 aortic dissections, 2 true aneurysms, 2 penetrating atherosclerotic ulcers, and 1 traumatic pseudoaneurysm.

Results - No complications resulting from the use of transesophageal echocardiography were observed. In 12 patients, the initial result was considered appropriate, with total or partial resolution of the major lesion confirmed by a posterior examination. In 1 patient, the procedure was suspended after transesophageal echocardiography and angiography showed that the proximal aortic diameter was inappropriate. Transesophageal echocardiography contributed to clarifying relevant points, such as aortic diameter, anatomic detail of the intimal lesion, and location and size of the communicating orifice. In addition, it facilitated placing the stent in the target lesion, reduced the time of exposure to radiation and the use of contrast medium, and provided rapid identification of intercurrent events, possibly reducing the total duration of the procedure.

Conclusion - The use of transesophageal echocardiography during placement of aortic stents seems appropriate. The actual advantages of the procedure will be defined in a comparative prospective study.

Keywords: transesophageal echocardiography, stents, aortopathies

Universidade Federal de São Paulo - Escola Paulista de Medicina

Mailing address: Cláudio Henrique Fischer - Av. Aclimação, 547/64 - 01531-001

- São Paulo, SP, Brazil - e-mail: c.h.fischer@uol.com.br

English version by Stela Maris C. e Gandour
Transesophageal echocardiography is considered a diagnostic method highly useful in diseases of the thoracic aorta, especially in aortic dissection, a situation in which it shows diagnostic accuracy similar to other methods, such as nuclear magnetic resonance, angiography, and helical computed tomography ${ }^{1-5}$. Compared with these examinations, transesophageal echocardiography has the advantage of allowing mobilization at the bedside, therefore enabling continuous monitoring of interventionist or surgical procedures performed in the thoracic aorta and in the proximal part of the abdominal aorta ${ }^{1,3,5-8}$. Recently, the appearance of self-expanding aortic endoprostheses (stents) has opened a new perspective in the therapeutical approach of selected cases of diseases of the descending thoracic aorta, particularly those with high surgical risk due to comorbidities ${ }^{9-13}$.

The objective of this study was to report our initial experience with transesophageal echocardiography used during implantation of self-expanding stents in the descending thoracic aorta at a hemodynamics laboratory.

\section{Methods}

From December' 97 to July '99, 27 consecutive patients with acute and chronic aortic diseases underwent implantation of aortic prostheses at the interventionist cardiology service at our institution. Out of this total, 13 patients (mean age of 54 years; 10 males) had the assistance of transesophageal echocardiography during the entire procedure, both for monitoring and helping to make decisions, constituting the case series of our study. Indications for stent implantation in this subgroup of patients included 8 aortic dissections of Stanford type B ( 2 acute cases, 1 of which was complicated with ischemia of the lower limbs), 2 true aneurysms of the descending aorta, 2 penetrating atherosclerotic ulcers, and 1 traumatic pseudoaneurysm in the isthmic region, partially corrected by surgery in the acute phase.

Transesophageal echocardiography was performed 
with the Apogee and Ultramark 9 HDI devices of the ATL brand (Advanced Technology Laboratories Inc., Bothel, USA) and multiplane and biplane esophageal probes of 5 $\mathrm{MHz}$, respectively, using techniques and sections previously established for obtaining aortic images ${ }^{14,15}$, with the patient in the dorsal decubitus position. All patients underwent general anesthesia and mechanical ventilation. At the beginning of the procedure, transesophageal echocardiography was used for diagnostic confirmation and catheterization of anatomical aspects that could contribute in choosing the dimensions of the endoprosthesis and its better position. Echocardiographic images of the aorta were continuously monitored during stent deployment to allow immediate identification of eventual complications, as well as immediate assessment of the stent's expansion, position, and flow pattern in its interior.

Endoprostheses were introduced through right or left femoral access by surgical isolation of the artery, according to the previous angiography, which defined the best side to be used ${ }^{10,13}$. Angiographic assessments during the procedure were performed with images obtained after injecting contrast medium through a catheter inserted by brachial or femoral via in the opposite side of the prosthesis access. Ten minutes after expansion of the endoprosthesis, a last injection was performed for a definite angiographic assessment of the procedure.

\section{Results}

Transesophageal echocardiography played an active role in all stent implantations, and no complication resulting from its use or from general anesthesia occurred. In all patients, the transesophageal echocardiography identified the target lesion and provided, therefore, monitoring of all procedures that precede stent deployment, especially identifying the catheters and following their position.

In 12 patients, the anatomic aspect was favorable for stent implantation, according to both angiography and transesophageal echocardiography. Only in the patient with a true aneurysm of the proximal descending aorta, did the procedure have to be interrupted. In this patient, transesophageal echocardiography confirmed the dimensions of the proximal neck of the aorta, in which the endoprosthesis was going to be implanted, obtained by angiography. By that time, these dimensions proved to be inadequate, ie, they were greater than those of the largest endoprosthesis available, which made the resolution of the target lesion impossible. In this case, surgical correction of the aneurysm was chosen as the best management.

In 10 patients, just 1 stent sufficed for repairing the target lesion, and this repair was considered total in 7 patients (fig. 1). In the other 3 patients, the result was considered appropriate, but with partial resolution of the major lesion, in which a residual flow persisted between the stent and the wall of the aorta in its proximal portion, according to the angiographic and transesophageal echocardiographic findings (fig. 2). Two patients required placement of a second stent for resolution of the target lesion. One of these patients had an extensive true aneurysm in the descending aorta, and the surgical treatment was contraindicated due to clinical complications. Even after implanting the second stent, the residual flow persisted through the false lumen created between the stents and the wall of the aneurysm, and this was shown by transesophageal echocardiography. The other patient with aortic dissection treated initially with stent

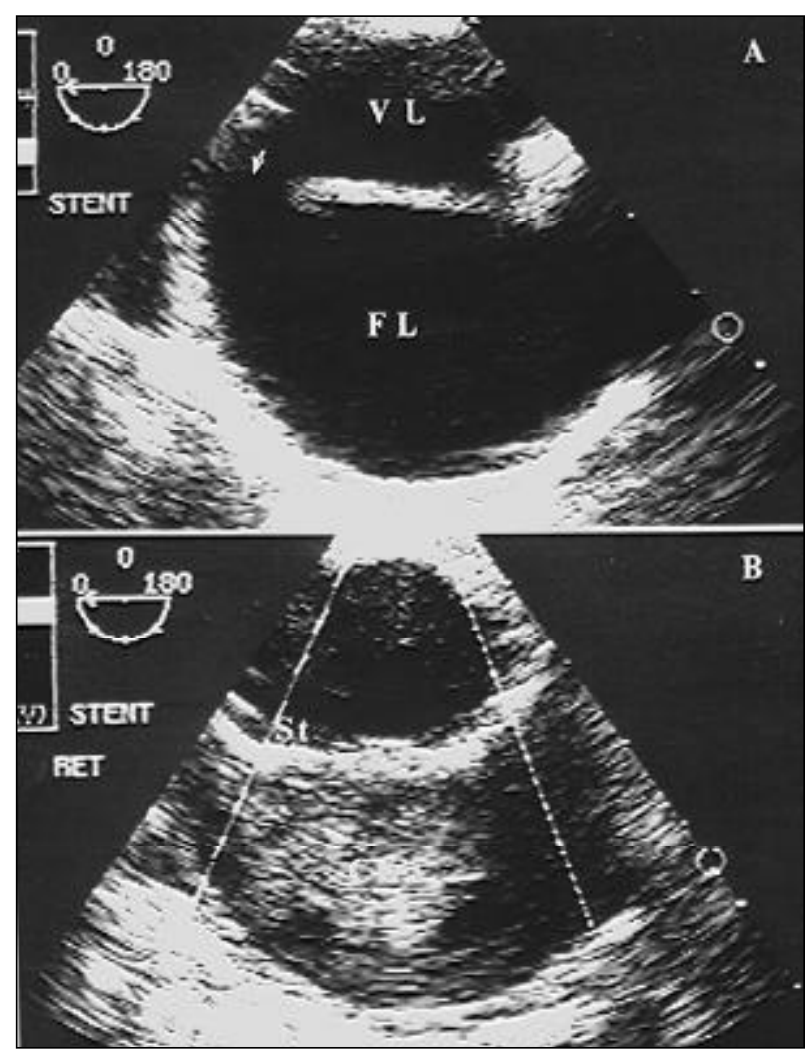

Fig. 1 - A) Type B aortic dissection with a wide communicating orifice (arrow) and false lumen (FL) with no signs of thrombosis; B) after stent deployment (St), closure of the communicating orifice and presence of intense spontaneous contrast in the false lumen suggesting thrombosis in progression. $\mathrm{VL}$ - true lumen.

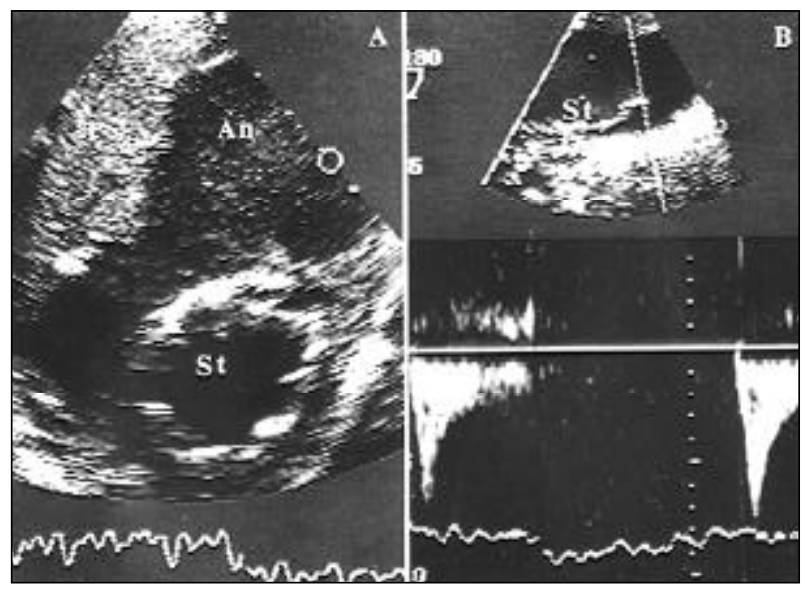

Fig. 2 - A) Stent implantation (St) in an aneurysm (An) of the descending aorta with mural thrombosis (T) and spontaneous contrast in the excluded portion; B) small residual flow between the stent and the aortic wall. 
placement in the proximal portion of the descending aorta without the aid of transesophageal echocardiography still had a persistent flow through the false lumen with compression of the true lumen in the thoracoabdominal transition. After placement of a new stent in this site, a reduction in flow obstruction was noted, but a new communicating orifice opened in the proximal extremity of the stent, which was promptly identified with transesophageal echocardiography. Placement of a third and last stent allowed continuity between the formerly and the latterly implanted stents, resulting in total resolution of the lesion. No neurological complication due to the presence of this set of 3 sequential stents occurred.

In 2 patients, the procedure was performed on an emergency basis. One of these patients had aortic dissection of type B complicated with ischemia of the lower limbs. In this case, the stent placement was imprecise without improvement in the ischemia, and the patient evolved with progressive clinical worsening and no condition for surgery, dying in 2 days. The other patient had an excellent outcome, with total resolution of the target lesion. This and the remaining 10 patients were discharged from the hospital in a mean period of 12 days.

\section{Discussion}

Endoprostheses have been used so far in the treatment of aortic diseases located in the descending portion of the aorta (true aneurysms, traumatic pseudoaneurysms, penetrating ulcers, and, more recently, type B aortic dissections ${ }^{9-13}$. Technical difficulties do not allow their use in the aortic arch and the ascending aorta, basically due to the aortic bending in these portions and the emergence of essential vessels that cannot be occluded, except for the involvement of the distal portion of the arch ${ }^{12}$. Recently, new endoprostheses have been developed and used to bypass this situation ${ }^{16}$.

Implantation of endoprostheses has been performed in different types of lesions of the descending aorta, as long as a proximal and a distal neck to the lesion may be delimitated. These necks should have diameters not larger than that of the largest endoprosthesis available. This limitation may occur when the rest of the aorta is dilated, frequently in the proximal neck, making the procedure unfeasible or jeopardizing the final result. This type of assessment is routinely performed based on previous computed tomography or angiography; it, however, may be successfully carried out with transesophageal echocardiography, as was clearly shown in a patient in our series. Nevertheless, even partial resolution of the aneurysm with persistence of a residual flow seems beneficial medium-term and may even change the natural evolution of the disease. Aneurysms that were not completely occluded by stent placement showed a better prognosis in 2 years, with a lower growth rate, if present, or even a reduction in their caliber ${ }^{17}$.

In the case of aortic dissections, location and extension of the communicating orifice and the fragility of the in- tima before and after the dissection, which may be well characterized on transesophageal echocardiography, seem to influence the approach and the result. After stent deployment and closure of the target orifice, new orifices may appear close to the extremities of the endoprosthesis. These new orifices may be promptly recognized on transesophageal echocardiography, and implantation of a second endoprosthesis may be required, as happened in 1 patient in our series, or the orifices may impair the late results due to maintenance of flow in the false lumen. This situation differs from maintenance of a small residual flow in the false lumen through a communication between the aortic wall and the stent, due to incomplete accommodation of the stent, as was identified in 3 cases in our series of transesophageal echocardiography. In this situation, the prognosis is favorable because the residual flow usually tends to disappear and, consequently, total thrombosis of the affected region develops, which may be shown on subsequent echocardiographic, angiographic, and tomographic studies ${ }^{11,8,19}$.

Penetrating ulcers, when involving the aorta in a localized manner and with no associated aneurysm, tend to have more favorable results, because the endoprosthesis is anchored in a theoretically healthy aortic wall, which has a satisfactory diameter both in its proximal and distal extremities.

We had only 1 patient with traumatic pseudoaneurysm in our series, and the transesophageal echocardiography was valuable in demonstrating the difficulty for total expansion of the first segment of the endoprosthesis due to bending of the distal portion of the aortic arch. The percutaneous aortic endoprosthesis monitored on transesophageal echocardiography may be a good alternative for patients with traumatic pseudoaneurysm of the aorta and who cannot undergo thoracic surgery. A larger experience with this procedure in this infrequent situation is, however, required.

Transesophageal echocardiography has not been routinely used during aortic stent placement in all institutions performing this technique, maybe due to elevation in costs or operational difficulties. Use of transesophageal echocardiography, however, seems necessary to guarantee better results, as shown in the study by Nienaber et al ${ }^{20}$, in which the authors observed total thrombosis of the false lumen and total occlusion of the communicating orifice in all patients with type B aortic dissection, who underwent stent implantation. In other studies ${ }^{21,22}$, in which no transesophageal echocardiography was used during the procedure, the results were not totally satisfactory, even though differences in the structure of the endoprosthesis and in the technique of implantation, which is constantly being perfected, may make this type of analysis difficult ${ }^{19,23}$. In our study, transesophageal echocardiography contributed to clarify relevant points for aortic endoprosthesis implantation, such as aortic diameter at the site of the lesion, diameters of the proximal and distal necks, anatomic aspect of the intimal lesion, location and size of the communicating orifice, and identification of true and false lumens for correct positioning of the catheters and guidewire. In addition, transesophageal 
echocardiography made stent placement in the target lesion easier, monitoring the position of catheters and stent deployment, reducing the need for angiographic images, therefore reducing the duration of exposure to radiation and contrast medium use. It also enabled rapid identification of intercurrent events, and, probably reduced the total duration of the procedure.

The auxiliary role played by transesophageal echocardiography becomes more evident in situations in which the use of radiographic contrast medium is not advised, as happened with 2 patients with impairment of the renal function in our series. In these patients, the stent was placed basically guided by transesophageal echocardiography. This fact draws attention to the possibility of using transesophageal echocardiography for orienting the implantation of aortic endoprostheses through surgical via in an isolated manner, eliminating the use of radioscopy and making the procedure easier ${ }^{13}$.

Expansion of this limited series of patients is required for definitely establishing the role played by transesophageal echocardiography in the percutaneous treatment of diseases of the descending aorta.

\section{References}

1. Wiet SP, Pearce WH, McCarthy WJ, Joob AW, Yao JST, McPherson DD. Utility of transesophageal echocardiography in the diagnosis of disease of the thoracic aorta. J Vasc Surg 1994; 20: 613-20.

2. Erbel R, Engberding R, Daniel W, et al. Echocardiography in diagnosis of aortic dissection. Lancet 1989; 1: 457-61.

3. Ballal RS, Nanda NC, Gatewood R, et al. Usefulness of transesophageal echocardiography in assessment of aortic dissection. Circulation 1991; 84: 1903-14.

4. Blanchard, Kimura BJ, Dittrich HC, DeMaria NA. Transesophageal echocardiography of the aorta. JAMA 1994; 272: 546-51.

5. Campos $\mathrm{F}^{\circ} \mathrm{O}$. Ecocardiografia transesofágica. Indicações e impacto clínico. Arq Bras Cardiol 1995; 64: 383-9.

6. Troianos CA, Savino JS, Weiss RL. Transesophageal echocardiographic diagnosis of aortic dissection during cardiac surgery. Anesthesiology 1991; 75: 149-53.

7. Epperlein S, Mohr-Kahaly S, Erbel R, Kearney P, Meyer J. Aorta and aortic valve morphologies predisposing to aortic dissection. An in vivo assessment with transesophageal echocardiography. Eur Heart J 1994; 15: 1520-7.

8. Simon P, Owen AN, Havel M, et al. Transesophageal echocardiography in the emergency surgical management of patients with aortic dissection. J Thorac Cardiovasc Surg 1992; 103: 1113-8.

9. Palma JH, Carvalho AC, Buffolo E, Almeida DR, Gomes WJ, Brasil LA. Endoscopic placement of stents in aneurysms of the descending thoracic aorta. Ann Thorac Surg 1998; 66: 256-8.

10. Fonseca JHP, Buffolo E, Carvalho AC, et al. Utilização de endoprótese autoexpansível (stent) introduzida através da artéria femoral para tratamento de dissecção da aorta descendente. Arq Bras Cardiol 1998; 70: 389-92.

11. Dake MD, Miller DG, Semba CP, Mitchell RS, Walker PJ, Liddell RP. Transluminal placement of endovascular stent-grafts for the treatment of descending thoracic aortic aneurysms. N Engl J Med 1994; 331: 1729-34.

12. Mitchell RS, Dake MD, Semba CP, et al. Endovascular stent-graft repair of thoracic aortic aneurysms. J Thorac Cardiovasc Surg 1996; 111: 1054-62.
13. Fonseca JHAP. Tratamento das dissecções aórticas agudas da aorta descendente utilizando stents aórticos introduzidos pela artéria femoral. Tese Livre-Docência - Escola Paulista de Medicina, Universidade Federal de São Paulo, São Paulo, 1999.

14. Seward JB, Khandheria BK, Oh JK, et al. transesophageal echocardiography: technique, anatomic correlations, implementation, and clinical applications. Mayo Clin Proc 1988; 63: 649-80.

15. Seward JB, Khandheria BK, Freeman WK, et al. Multiplane transesophageal echocardiography: image orientation, examination technique, anatomic correlations, and clinical applications. Mayo Clin Proc 1993; 68: 523-51.

16. Inoue $\mathrm{K}$, Hosokawa $\mathrm{H}$, Iwase $\mathrm{T}$, et al. Aortic arch reconstruction by transluminally placed endovascular branched stent graft. Circulation 1999; 100(19suppl): II316-21.

17. Matsumura JS, Moore WS. Clinical consequences of periprosthetic leak after endovascular repair of abdominal aortic aneurysm. J Vasc Surg 1998; 27: 606-13.

18. Parodi JC, Palmaz JC, Barone HD. Transfemoral intraluminal graft implantation for abdominal aortic aneurysms. Ann Vasc Surg 1991; 5: 491-9.

19. Dake MD, Miller DC, Mitchell RS, Semba CP, Moore KA, Sakai T. The "first generation" of endovascular stent-grafts for patients with aneurysms of the descending thoracic aorta. J Thorac Cardiovasc Surg 1998; 116: 689-704.

20. Nienaber CA, Fattori R, Lund G, et al. Nonsurgical reconstruction of thoracic aortic dissection by stent-graft placement. N Engl J Med 1999; 340: 1539-45.

21. Dake MD, Kato N, Mitchell RS, et al. Endovascular stent-graft placement for the treatment of acute aortic dissection. N Engl J Med 1999; 340: 1546-52.

22. Kato M, Matsuda T, Kaneko M, et al. Outcomes of stent-graft treatment of false lumen in aortic dissection. Circulation 1998; 98: II-305-12.

23. Mitchell RS, Miller DC, Dake MD, Semba CP, Moore KA, Sakai T. Thoracic aortic aneurysm repair with an endovascular stent graft: the "first generation". Ann Thorac Surg 1999; 67:1971-4. 